\title{
Two-Parameters Bifurcation in Quasilinear Differential-Algebraic Equations
}

\author{
Kamal .H .Yasir \& Abbas. Mohsen. Alhusenawe \\ University of Thi- Qar The College of Education Sciences Pure \\ Department of mathematics
}

\section{ABSTRACT}

In this paper, bifurcation of solution of guasilinear quasilinear DAE is eventually reducible to an ordinary differential equati on (ODEs) and that this reduction so we canapply the classical bifurcation theory of the (ODEs). The taylor expansion appl ied tothe reduced DAEs to prove that is equivalent to an ODE which is a normal form undersome non degeneracy Conditions

theorems given in this work deal with the saddle node,transcritical and pitchfork bifurcation with twoparameters. Some illus trated examples are given to explain the idea of the paper.

\section{Keywords : Differential Algebraic Equation ; Quasilinear; Bifurcation. \\ INTRODUCTION}

Nearly all DAEs arising in scientific or engineering problems are quasilinear. Thisarticle presents bifurcationin guasilinear di fferentialalgebraic equations (DAEs) differfrom ordinary differential equations (ODEs).Over the years several approaches havebeen introduced for the study of local existence and uniquenessquestions for DAEs. While they exhibit major technical differences and are based on different assumptions,all these approaches agreewith the basic principle that a DAE is even tually reducibleto an ODE and that this reduction should be donevia a recursiveprocess. The bifurcation in guasilinear para meterized DAEs form

$$
A(\mu, x) \dot{x}=G(\mu, x), \quad \mu \in \mathrm{R}^{2}, \mathrm{x} \in \mathrm{R}^{1},
$$

it will be investigated. Accordingly, we shall assume that for some open interval $I \subset$ Rand open subset $U \mathrm{n} \subset \mathrm{Rn}$

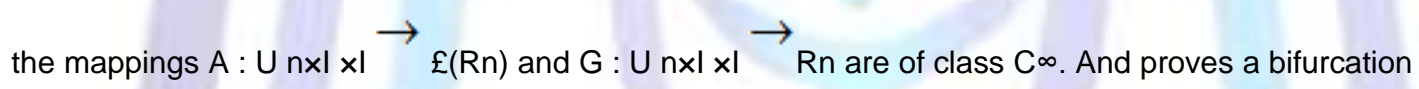

theorem based on assumptions on theTaylor coefficients. Since we will impose conditions on these coefficients we will be ableto show that the system undergoes saddle node ,transcritical and pitchfork bifurcationthat is a little more akin to bifurcation ODE .

A simple comparison of the areas of the sciences in which DAEs are involved withthose in which examples of bifurcation in ODEs arise [1],[3] that bifurcation of periodicsolutions occurs from (0,0)and [4] Our exposition is based on Jepson, A. and Spence[2] and the references therein reveals a considerable overlap and suggests that an appropriate variant of the bifurcationtheorem should be available in the DAE setting.it is important note that all theorems and condition $\mathrm{S}$

for Bifurcation to be occurredin the reduced DAEs will be given in terms of $A$ and $G$ in $(1,1)$ and thiswillbe extension of the bifurcation theory to DAEs of index one.

In the index one case, our coal is it use the reduction of (1.1) to an ODE In withthe reduction (1.1) method given in [5]

then apply classical bifurcation theory to the reduced ODEs.

This paper is organized as follows: Section 2 deals with the problem of reducingparameterized families of DAEs simultaneously.Since reduction of DAEs to ODE formleads to implicit rather than explicit ODEs, it is important to rephrase some of thehypotheses of the classical bifurcation theorems in that setting. This is done in section

3. The bifurcation theorems for quasilinear DAEs is proved in theorems $(3.1,3.2,3.3)$

(for two-parameters ) in Section 4 we will study of the behavior bifurcation 
and discusstheir implementation in Maple.

\section{Reduction of Parametrized DAEs [5]}

The bifurcation in guasilinear parameterized DAEs form (1.1) will be investigatedand DAEs will be reduced to an equivalent parameterized ODEs. Then classical bifurcation theory can be applied. In the reduction peroses we will follow the methodof reduction given in [5]. So the following theorem is an essential in our work, which summarized the reduction of DAEs (1.1).

\section{Theorem 2.1. [5]}

Let $(\check{X}, \breve{\mu}) \in \mathrm{W}_{1}$ and let $\Phi=\mathrm{id} \times \varphi: \epsilon \mathrm{IX|X} U^{r 1} \rightarrow \mathrm{IX} U^{n}$ be a local $C^{\infty}$ parametrization of $\mathrm{W}_{1}$ near $(\check{X}, \breve{\mu})$. There exist an open subinterval $\check{I}$ CI with $\breve{\mu} \in \mathbf{I} \times \mathbf{I}$ land an open neighborhood $O^{n} C U^{n}$ of $\check{x}$ with the following property :For $\mu \in \check{I}$ ,a $C^{\infty}$ mapping $x: J \rightarrow O^{n}$ on an open interval $J \subset R$ is a solution of the DAE (1.1) if and only if $x(t)=\phi(\mu, \xi(t)), \forall t \in J$, and $\xi: J \rightarrow U^{r 1}$ is a $C^{1}$ solution of the system $A_{1}(\mu, \xi) \xi=G_{1}(\mu, \xi)$, where $W_{1}=(x, \mu) \in U^{n} \times \mathbf{I}: G(x, \mu) \in \operatorname{rgeG}(x, \mu)$, and $A_{1}: I_{x} I_{x} U^{r 1} \rightarrow \Sigma\left(R^{r 1}, \operatorname{rgeA}(\check{\mu}, \ddot{x})\right)$ $\left.\cong \varepsilon\left(R^{r 1}\right), G_{1}: I \times I \times U^{r 1} \rightarrow \operatorname{rgeA}(\widetilde{\mu}, \widetilde{x})\right)\left(R^{r 1}\right)$ are the $C^{\infty}$ mapping given $b$

$$
\begin{aligned}
& \mathrm{A}_{1}(\mu, \xi):=P_{\mathrm{A}}(\mu, \phi(\mu, \xi)) \mathrm{D} \xi \phi(\mu, \xi), \\
& \mathrm{G}_{1}(\mu, \xi):=P_{\mathrm{G}}(\mu, \phi(\mu, \xi)),
\end{aligned}
$$

and $\mathscr{P}_{\in} \in\left(R^{\mathrm{n}}\right)$ is an arbitrary linear projection onto rge $\left.\mathrm{A}(\mu, \mathrm{x})\right) \stackrel{\cong}{=}\left(\mathrm{R}^{\mathrm{r} 1}\right)$. or Fixed $\mu \in \mathbf{I} \times \mathbf{I}$ and $x_{1}^{\mu} \in W_{1}^{\mu}$, the $\mathrm{DAE}$ (1.1) reduces to the form

$A_{1}(\mu, \xi) \xi=G_{1}(\mu, \xi)$

The fixed but arbitrary $\mu=(\mu 1, \mu 2) \in \mathbf{I} \times \mathbf{I}$ are also given by the solutions of thenon parametrized DAE

$$
A(\mu, x)\left(\frac{\tilde{N}}{\tilde{x}}\right)=G(\mu, x)
$$

where

$$
A=\left(\begin{array}{ll}
1 & 0 \\
0 & A
\end{array}\right), G=\left(\begin{array}{l}
0 \\
G
\end{array}\right)
$$

For the sake of argument, assume that the $\operatorname{DAE}(1.1)$ with $\mu=\check{\mu}_{\text {has }}$ index one at ${ }^{\mathscr{X}} \in W_{1}^{\mu}$ (so that $\left.(\check{\mu}, \mathbb{x}) \in W_{1}\right)$.

With the previous notation, this means that the operator $A_{1}(\widetilde{\mu}, \xi)$ where $\phi(\widetilde{\mu}, \xi)=\bar{x}$,

has full rank $r 1$ and hence is invertible.By continuity $A_{1}(\mu, \xi)$ remains invertible for $(\mu, \xi)$ near $(\breve{\mu}, \widetilde{X}) \in R \times R^{r 1}$ and it thus follows fromTheorem 2.1, that in the vicinity of $(\check{\mu}, \widetilde{X}) \in W_{1}$, the parameterized DAE (1.1) is equivalent to the explicit parameterized ODE:

$$
\xi=A_{1}(\mu, \xi)^{-1} G_{1}(\mu, \xi), \xi \in U^{r n}
$$

To motivate the discussion in the next section, suppose also $\ddot{x}=0, \breve{\mu}=0$

\section{Local two-Parameter Bifurcations of Equilibrium Points}

We will now consider the general guasilinear parametrized DAEs equation 
$A(\mu, x) \tilde{x}=G(\mu, x)$,

Journal of Advances in Mathematics $\mu \in \mathrm{R}^{2}, \mathrm{x} \in \mathrm{R}^{1}$,

and $\mu=\left(\mu 1, \mu_{2}\right)$, and prove a bifurcation theorems based on assumptions on the Taylorexpansion of $\mathrm{G}$. We assume that $G(x, \mu)$ for all value of $\mu, A(x, \mu)$ is independent of $x$ and $\mu$.

\subsection{Saddle-Node bifurcation}

The saddle-node bifurcation can take place in any system and is, in fact, a very typicalbifurcation to happen when a parameter is varied. Maybe because this bifurcation isso typical, it has a lot of other names. The saddle-node bifurcation is also called foldbifurcation, tangent bifurcation, limit point bifurcation, or turning point bifurcation. from theorem $(1.1)$ it follow that near $(0,0)$ that DAEs $(1.1)$ reduced to the system

$\xi=A_{1}(\mu, \xi)^{-1} G_{1}(\mu, \xi), \xi \in U^{r n}$. where $U^{r} \subset R^{r 1}$ is an open subset $A_{1}: I \times I \times U^{n} \rightarrow\left(f\left(R^{r 1}, \operatorname{reg} A(0,0)\right)\right.$ $\left.\approx\left(R^{n}\right)\right)$ and $G_{1}: I \times I \times U^{n} \rightarrow\left(f\left(R^{r 1}, \operatorname{reg} A(0,0)\right) \approx\left(R^{n}\right)\right)$ are of class $C^{\infty}$. the following theorem related to this kind of bifurcation.

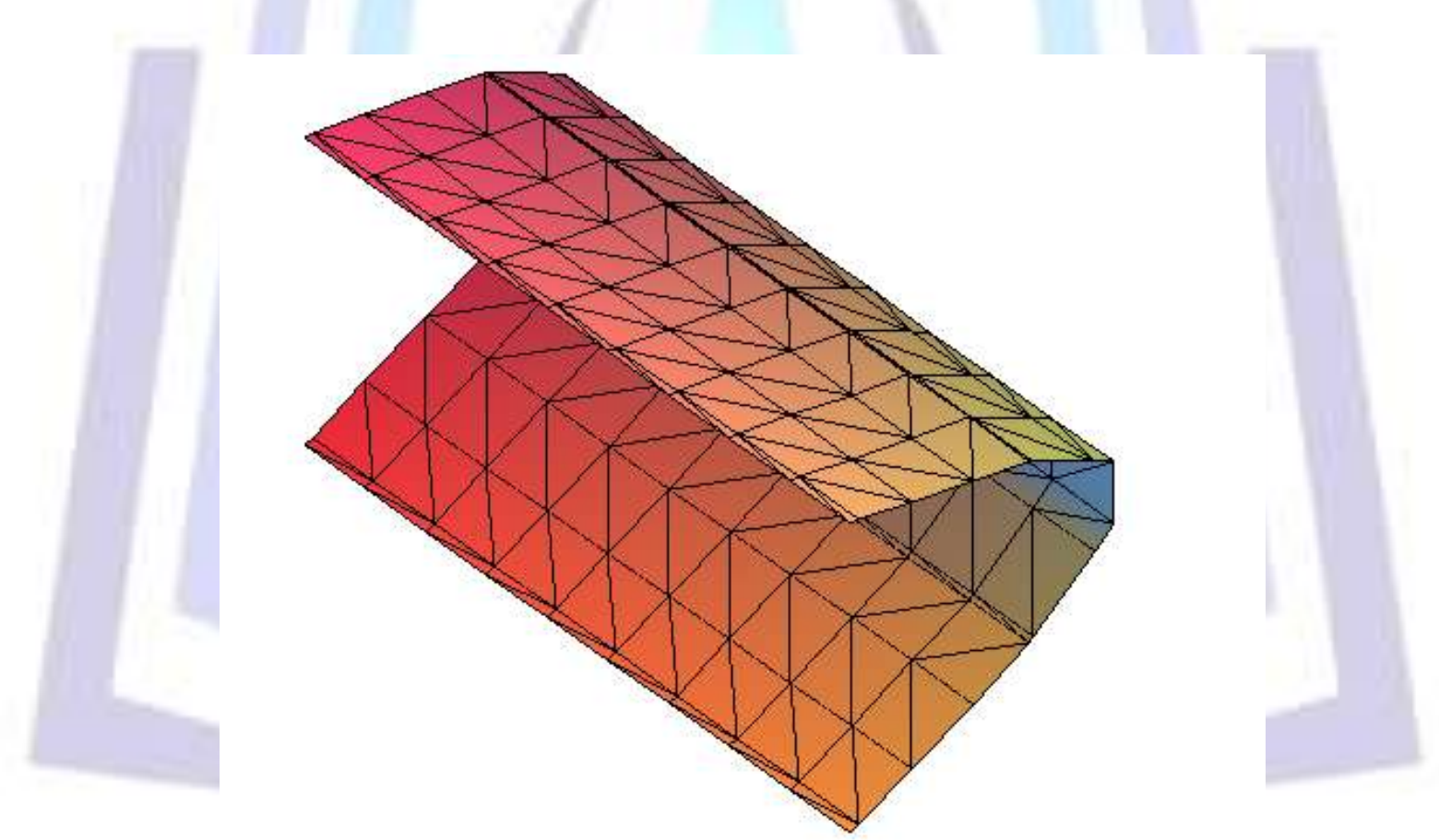

Figure 1: The normal form of a saddal- node bifurcation, where $r$ ranges from $\pi$ to- $\pi$ using mapleTheorem

Theorem 3.1. Consider one-dimensional quasilinear DAEs

$$
\mathrm{A}(\mathrm{x}, \mu) \dot{x}=\mathrm{G}(\mathrm{x}, \mu), \quad \mu \in \mathrm{R}^{2}, \mathrm{x} \in \mathrm{R}^{1}
$$

where $\mathrm{G} \in R^{3}$ has at $\mu=0$ the eguilibrium $x=0$, and $\frac{\partial G}{\partial x}(0,0,0) \neq 0$. Assume that the following two non-degeneracy conditions are satisfied:

(i) $\quad \frac{\partial^{2} G}{\partial \mathrm{x}^{2}}(0,0,0) \neq 0$

(ii) $\quad \frac{\partial G}{\partial \mu_{1}}(0,0,0) \neq 0 \& \frac{\partial G}{\partial \mu_{2}}(0,0,0) \neq 0$

then near $(0,0,0),(3.2)$ is topologically equivalent to the one of the following normalforms: 
Proof. According to the reduction processes mentioned in (Section 2) DAE will be reduced to ODEs:

$$
\xi=A_{1}(\xi, \mu)^{-1} G_{1}(\xi, \mu)
$$

where $G_{1}(\xi, \mu)$ and $A_{1}(\xi, \mu)^{-1}$ from theorem 2.1 reduced to $G(x, \mu)$ and $A(x, \mu)$. Then by Taylor expansion about $(0,0,0)$ we have:

$G_{1}\left(\xi, \mu_{1}, \mu_{2}\right)=G_{1}(0,0,0)+\frac{\partial G_{1}}{\partial \xi}(0,0,0) \xi+\frac{\partial G_{1}}{\partial \mu_{1}}(0,0,0) \mu_{1}+\frac{\partial G_{1}}{\partial \mu_{2}}(0,0,0) \mu_{2}+\frac{\partial^{2} G_{1}}{\partial \xi^{2}} \frac{\xi^{2}}{2}$

$\frac{\partial^{2} G_{1}}{\partial \xi \delta \mu_{1}} \xi \mu_{1}+\frac{\partial^{2} G_{1}}{\partial \xi \delta \mu_{2}} \xi \mu_{2}+\frac{\partial^{2} G_{1}}{\delta \mu_{1} \partial \mu_{2}} \mu_{1} \mu_{2}+\frac{\partial^{2} G_{1}}{\partial \mu_{1}{ }^{2}} \frac{\mu_{1}^{2}}{2}+\frac{\delta^{2} G_{1}}{\delta \mu_{2}^{2}} \frac{\mu_{2}^{2}}{2}+O\left(\mu_{1}, \mu_{2}, \xi\right)^{3}$

where.

$$
G_{1}(0,0,0)=0, \frac{\partial G_{1}}{\partial \xi}(0,0,0)=0, \quad \frac{\partial^{2} G_{1}}{\partial \xi^{2}} \neq 0
$$

Next we remove the linear term w.r.t $\xi$ by introducing a new variable $z$ :

$$
\xi=z+\delta \text {, }
$$

where $\delta$ is unknown parameter the inverse coordinate transformation is

$$
z=\xi-\delta \text {. }
$$

Differentiate the direct transformation (3.5) we get:

$\frac{d z}{d t}=A_{1}(z+\delta, 0,0)^{-1}\left[+\frac{\partial G_{1}}{\partial \mu_{1}} \mu_{1}+\frac{\partial G_{1}}{\partial \mu_{2}} \mu_{2}+\frac{\partial^{2} G_{1}}{\partial z^{2}} \frac{(\mathrm{z}+\xi)^{2}}{2}+\frac{\partial^{2} G_{1}}{\partial \mathrm{z} \delta \mu_{1}}(\mathrm{z}+\delta) \mu_{1}+\frac{\partial^{2} G_{1}}{\partial \mathrm{z} \delta \mu_{2}}(\mathrm{z}+\delta) \mu_{2}+\right.$ $\frac{\partial^{2} G_{1}}{\delta \mu_{1} \partial \mu_{2}} \mu_{1} \mu_{2}+\frac{\partial^{2} G_{1}}{\partial \mu_{1}^{2}} \frac{\mu_{1}^{2}}{2}+\frac{\delta^{2} G_{1}}{\delta \mu_{2}^{2}} \frac{\mu_{2}^{2}}{2}+O\left(\mu_{1}, \mu_{2}, \mathrm{z}+\right)^{3}$

\section{Therefore,}

$\frac{d z}{d t}=A_{1}(z+\delta, 0,0)^{-1}\left[\frac{\partial G_{1}}{\partial \mu_{1}} \mu_{1}+\frac{\partial^{2} G_{1}}{\partial \mu_{1}} \frac{\mu_{1}}{2}+\frac{\partial^{2} G_{1}}{\partial z \delta \mu_{1}} \delta \mu_{1}+\frac{1}{2} \frac{\partial^{2} G_{1}}{\delta \mu_{1} \partial \mu_{2}} \mu_{1} \mu_{2}+\frac{1}{2} \frac{\partial^{2} G_{1}}{\partial z^{2}} \frac{\delta^{2}}{2}\right.$

$+z\left(\frac{\partial^{2} G_{1}}{\partial z \delta \mu_{1}} \delta \mu_{1}+\frac{1}{2} \frac{\partial^{2} G_{1}}{\partial z^{2}} \delta\right)+\frac{1}{4} \frac{\partial^{2} G_{1}}{\partial z^{2}} z^{2}+\frac{\partial G_{1}}{\partial \mu_{2}} \mu_{2}+\frac{\partial^{2} G_{1}}{\partial \mu_{2}} \frac{\mu_{2}{ }^{2}}{2}+\frac{\partial^{2} G_{1}}{\partial z \delta \mu_{2}} \delta \mu_{2}+$

$\left.\frac{1}{2} \frac{\partial^{2} G_{1}}{\delta \mu_{1} \partial \mu_{2}} \mu_{1} \mu_{2}+\frac{1}{2} \frac{\partial^{2} G_{1}}{\partial \mathrm{z}^{2}} \frac{\delta^{\mathrm{z}}}{2}+z\left(\frac{\partial^{2} G_{1}}{\partial \mathrm{z} \delta \mu_{2}} \delta \mu_{2}+\frac{1}{2} \frac{\partial^{\mathrm{n}} G_{1}}{\partial \mathrm{z}^{\mathrm{z}}} \delta\right)+\frac{1}{4} \frac{\partial^{2} G_{1}}{\partial \mathrm{z}^{\mathrm{z}}} z^{2}\right] \ldots$

by removing the linear terms $z\left(\frac{\partial^{2} G_{1}}{\partial \mathrm{z} \delta \mu_{1}} \delta \mu_{1}+\frac{1}{2} \frac{\partial^{2} G_{1}}{\partial z^{2}} \delta\right)$ and $z\left(\frac{\partial^{2} G_{1}}{\partial z^{2} \mu_{2}} \delta \mu_{2}+\frac{1}{2} \frac{\partial^{2} G_{1}}{\partial z^{2}} \delta\right)$ which is

required that:

$\delta_{1}(\mu)=-\frac{\frac{\partial^{2} G_{1}}{\partial z \delta \mu_{1}} \delta}{\frac{\partial^{2} G_{1}}{\partial z^{2}}} \mu_{1}$ and $\delta_{1}(\mu)=-\frac{\frac{\partial^{2} G_{1}}{\partial z \delta \mu_{2}} \delta}{\frac{\partial^{2} G_{1}}{\partial z^{2}}} \mu_{2}$.

Then the equation becomes:

$\frac{d z}{d t}=\beta_{1}+\beta_{2}+\frac{1}{2} \frac{\partial^{2} G_{1}}{\partial z^{2}} z^{2}$

Where 
$\beta_{1}=\frac{\partial G_{1}}{\partial \mu_{1}} \mu_{1}+\frac{\partial^{2} G_{1}}{\partial \mu_{1}^{2}} \frac{\mu_{1}^{2}}{2}+\frac{\partial^{2} G_{1}}{\partial z \delta \mu_{1}} \delta \mu_{1}+\frac{1}{2} \frac{\partial^{2} G_{1}}{\partial z^{2}} \frac{\delta^{2}}{2}$

$\beta_{2}=\frac{\partial G_{1}}{\partial \mu_{2}} \mu_{2}+\frac{\partial^{2} G_{1}}{\partial \mu_{2}} \frac{\mu_{2}^{2}}{2}+\frac{\partial^{2} G_{1}}{\partial \mathrm{z} \delta \mu_{2}} \delta \mu_{2}+\frac{1}{2} \frac{\partial^{2} G_{1}}{\partial \mathrm{z}^{2}} \frac{\delta^{2}}{2}$.

Now consider as a new parameter $\beta=\left(\beta_{1}(\mu), \beta_{2}(\mu)\right)$ and we have $A_{1}(z+\delta, 0,0)^{-1}$

is independent of $\xi$ and since $\frac{\partial G}{\delta \mu_{1}} \neq 0 \& \frac{\partial G}{\delta \mu_{1}} \neq 0$ we can neglecting terms with

$\mu_{1}^{2} \& \mu_{2}^{2}$ respectively then we have:

$\beta_{1} \approx \frac{\partial G_{1}}{\partial \mu_{1}} \mu_{1} \quad \& \quad \beta_{2} \approx \frac{\partial G_{1}}{\partial \mu_{2}} \mu_{2}$

So for $\mu_{1}>0, \frac{\partial G_{1}}{\partial \mu_{1}}>0, \beta_{1}$ is increasing and $\mu_{2}>0, \frac{\partial G_{1}}{\partial \mu_{2}}>0, \beta_{2}$ is increasing,

but if $\frac{\partial G_{1}}{\partial \mu_{1}}<0 \& \frac{\partial G_{1}}{\partial \mu_{2}}<0$ then $\beta_{1}, \beta_{2}$ are decreasing when $\mu_{1}<0, \mu_{1}<0$ resp. Now let the following assumption

$\gamma_{1}=\left\{\begin{array}{l}\beta_{1} \text { if } \frac{\partial G_{1}}{\partial \mu_{1}}>0 \\ \beta_{1} \text { if } \frac{\partial G_{1}}{\partial \mu_{1}}<0\end{array} \gamma_{1}=\left\{\begin{array}{l}\beta_{2} \text { if } \frac{\partial G_{1}}{\partial \mu_{2}}>0 \\ \beta_{2} \text { if } \frac{\partial G_{1}}{\partial \mu_{2}}<0\end{array}\right.\right.$

Then the equation is:

$\frac{d z}{d t} \pm \gamma_{1}^{+} \gamma_{2} \pm \frac{1}{2} \frac{\partial^{2} G_{1}}{\partial^{2}} z^{2}$.

Next assume $y=\left|\frac{1}{2} \frac{\partial^{2} G_{1}}{\partial z^{2}}\right| z$ then we have:

$\frac{d y}{d t}=\left|\frac{1}{2} \frac{\partial^{2} G_{1}}{\partial z^{2}}\right| \frac{1 d z}{2 d t}= \pm \gamma_{1}\left|\frac{1}{2} \frac{\partial^{2} G_{1}}{\partial z^{2}}\right| \pm \gamma_{2}\left|\frac{1}{2} \frac{\partial^{2} G_{1}}{\partial z^{2}}\right|+\left|\frac{1}{2} \frac{\partial^{2} G_{1}}{\partial z^{2}}\right| \frac{1}{2} \frac{\partial^{2} G_{1}}{\partial z^{2}} z^{2}$.

Substituting $z=\frac{y}{\left|\frac{1 a^{2} G_{1}}{2 a z^{2}}\right|}$ we get $\quad \frac{d y}{d t}= \pm \gamma_{1}\left|\frac{1}{2} \frac{\partial^{2} G_{1}}{\partial z^{2}}\right| \pm \gamma_{2}\left|\frac{1}{2} \frac{\partial^{2} G_{1}}{\partial z^{2}}\right|+\frac{\frac{1 \partial^{2} G_{1}}{a^{2}}}{\frac{1}{2} \mid \frac{\partial^{2} G_{1}}{a z^{2}} y^{2}}$.

Suppose that $\alpha_{1}=\gamma_{1}\left|\frac{1}{2} \frac{\partial^{2} G_{1}}{\partial z^{2}}\right|$ and $\alpha_{2}=\gamma_{2}\left|\frac{1}{2} \frac{\partial^{2} G_{1}}{\partial z^{2}}\right|$ Then we get the normal form

$\ddot{y}= \pm \alpha_{1} \pm \alpha_{2}+y^{2}+\mathrm{O}\left(y^{3}\right)$

\subsection{Trans-critical bifurcation}

If two curves of fixed points intersect at the origin in the $\mu-x$ plain, both existed on either side of $\mu=0$ then the origin is called a transcritical bifurcation (TCB) point see[8]. 


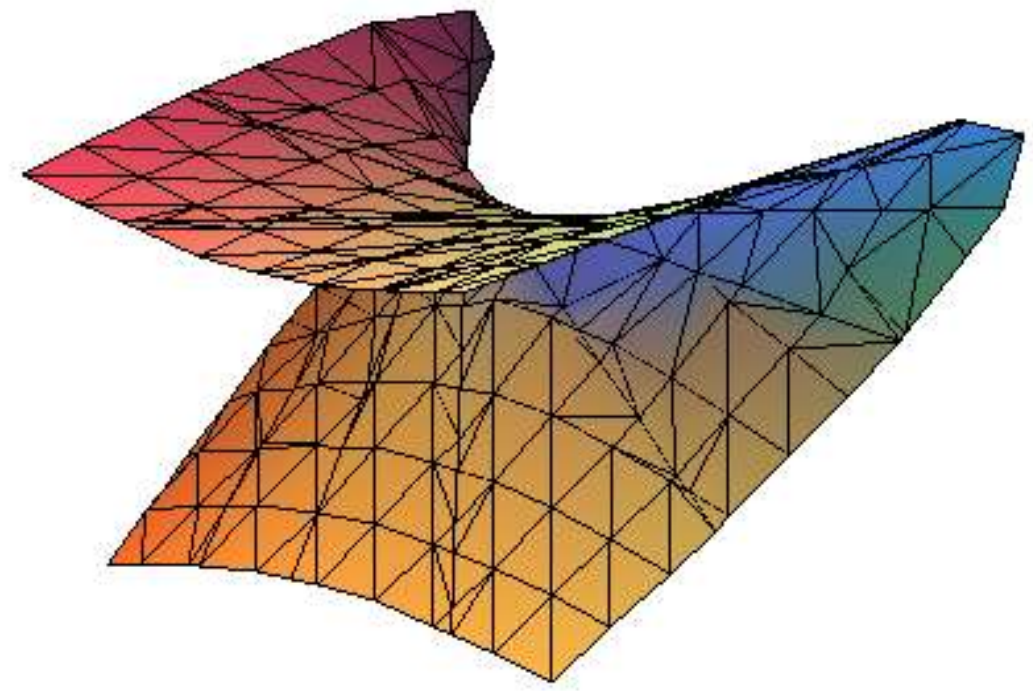

Figure 2: The normal form of a Trans-critical bifurcation, where $r$ ranges from $\pi$ to $-\pi$ using mapleTheorem

Theorem 3.2. Consider one-dimensional quasilinear DAEs

$$
\mathrm{A}(\mathrm{x}, \mu) \dot{x}=\mathrm{G}(\mathrm{x}, \mu), \quad \mu \in \mathrm{R}^{2}, \mathrm{x} \in \mathrm{R}^{1}
$$

where $\mathrm{G} \in R^{3}$ has at $\mu=0$ the eguilibrium $x=0$, and $\frac{\partial G}{\partial x}(0,0,0) \neq 0$.Assume that the following two non-degeneracy conditions are satisfied::
(i) $\quad \frac{\partial^{2} G}{\partial \mathrm{x}^{2}}(0,0,0) \neq 0$
(ii) $\frac{\partial^{2} G_{1}}{\partial x \partial \mu_{1}}(0,0,0) \neq 0 \& \frac{\partial^{2} G_{1}}{\partial x \partial \mu_{2}}(0,0,0) \neq 0$

then near $(0,0,0),(3.2)$ is topologically equivalent to the one of the following normalforms: $\dot{y}= \pm \alpha_{1} \mathrm{y} \pm \alpha_{2} \mathrm{y} \pm y^{2}+\mathrm{o}\left(y^{3}\right)$

Proof. According to the reduction processes mentioned in (Section 2) DAE will be reduced to ODEs:

$$
\xi=A_{1}(\xi, \mu)^{-1} G_{1}(\xi, \mu)
$$

where $G_{1}(\xi, \mu)$ and $A_{1}(\xi, \mu)^{-1}$ from theorem 2.1 reduced to $G(x, \mu)$ and $A(x, \mu)$. Then by Taylor expansion about $(0,0,0)$ we have:

$G_{1}\left(\xi, \mu_{1}, \mu_{2}\right)=G_{1}(0,0,0)+\frac{\partial G_{1}}{\partial \xi}(0,0,0) \xi+\frac{\partial G_{1}}{\partial \mu_{1}}(0,0,0) \mu_{1}+\frac{\partial G_{1}}{\partial \mu_{2}}(0,0,0) \mu_{2}+\frac{\partial^{2} G_{1}}{\partial \xi^{2}} \frac{\xi^{2}}{2}+$ $\frac{\partial^{2} G_{1}}{\partial \xi \delta \mu_{1}} \xi \mu_{1}+\frac{\partial^{2} G_{1}}{\partial \xi \delta \mu_{2}} \xi \mu_{2}+\frac{\partial^{2} G_{1}}{\delta \mu_{1} \partial \mu_{2}} \mu_{1} \mu_{2}+\frac{\partial^{2} G_{1}}{\partial \mu_{1}{ }^{2}} \frac{\mu_{1}{ }^{2}}{2}+\frac{\delta^{2} G_{1}}{\delta \mu_{2}^{2}} \frac{\mu_{2}{ }^{2}}{2}+O\left(\mu_{1}, \mu_{2}, \xi\right)^{3}$

where.

$$
G_{1}(0,0,0)=0, \frac{\partial G_{1}}{\partial \xi}(0,0,0)=0, \quad \frac{\partial^{2} G_{1}}{\partial \xi^{2}} \neq 0
$$

As in the proof of Theorem (3.1) we get: 
$\delta_{1}(\mu)=-\frac{\frac{a^{2} G_{1}}{\partial z \delta \mu_{1}} \delta}{\frac{a^{2} G_{1}}{a z^{2}}} \mu_{1}$ and $\delta_{1}(\mu)=-\frac{\frac{a^{2} G_{1}}{\partial z \delta \mu_{z}} \delta}{\frac{a^{2} G_{1}}{a z^{2}}} \mu_{2}$.

Journal of Advances in Mathematics

Then the equation becomes:

$\frac{d z}{d t}=\beta_{1}+\beta_{2}+\frac{1}{2} \frac{\partial^{2} G_{1}}{\partial z^{2}} z^{2}$

Where

$\beta_{1}=\frac{\partial^{2} G_{1}}{\partial \mathrm{z} \delta \mu_{1}} \delta \mu_{1}+\frac{1}{2} \frac{\partial^{2} G_{1}}{\partial \mathrm{z}^{2}} \delta$

$\beta_{2}=\frac{\partial^{2} G_{1}}{\partial z \delta \mu_{2}} \delta \mu_{2}+\frac{1}{2} \frac{\partial^{2} G_{1}}{\partial \mathrm{z}^{2}} \delta$.

Now consider as a new parameter $\beta=\left(\beta_{1}(\mu), \beta_{2}(\mu)\right)$ and we have $A_{1}(z+\delta, 0,0)^{-1}$

is independent of $\xi$ and since $\frac{\partial G}{\delta \mu_{1}} \neq 0 \& \frac{\partial G}{\delta \mu_{1}} \neq 0$ we can neglecting terms with $\mu_{1}^{2} \&_{\mu_{2}}^{2}$ respectively then we have:

$\beta_{1} \approx \frac{\partial^{2} G_{1}}{\partial z \delta \mu_{1}} \mu_{1} \beta_{2} \approx \frac{\partial^{2} G_{1}}{\partial z \delta \mu_{1}} \mu_{2}$

So for $\mu_{1}>0, \frac{\partial^{2} G_{1}}{\partial \mathrm{z} \delta \mu_{1}}>0, \beta_{1} \quad$ is increasing and $\mu_{2}>0, \& \frac{\partial^{2} G_{1}}{\partial \mathrm{z} \delta \mu_{2}}>0, \beta_{2}$ is increasing, but if $\frac{\partial^{2} G_{1}}{\partial \mathrm{z} \delta \mu_{1}}<0 \& \frac{\partial^{2} G_{1}}{\partial \mathrm{z} \delta \mu_{2}}$ then $\beta_{1}, \beta_{2}$ are decreasing when $\mu_{1}<0, \mu_{1}<0$ resp. Now let the following assumption

$\gamma_{1}=\left\{\begin{array}{l}\beta_{1} \text { if } \frac{\partial^{2} G_{1}}{\partial \mathrm{z} \delta \mu_{1}}>0 \\ \beta_{1} \text { if } \frac{\partial^{2} G_{1}}{\partial \mathrm{z} \delta \mu_{1}}<0\end{array} \gamma_{1}=\left\{\begin{array}{l}\beta_{2} \text { if } \frac{\partial^{2} G_{1}}{\partial \mathrm{z} \delta \mu_{2}}>0 \\ \beta_{2} \text { if } \frac{\partial^{2} G_{1}}{\partial \mathrm{z} \delta \mu_{2}}<0\end{array}\right.\right.$

Then the equation is:

$\frac{d z}{d t}= \pm \gamma_{1} \frac{\partial^{2} G_{1}}{\partial z^{2}} \gamma_{2} \frac{\partial^{2} G_{1}}{\partial z^{2}} z \pm \frac{1}{2} \frac{\partial^{2} G_{1}}{\partial z^{2}} z^{2}$

Next assume $y=\left|\frac{1}{2} \frac{\partial^{2} G_{1}}{\partial z^{2}}\right| z$ then we have:

$\frac{d y}{d t}=\left|\frac{1}{2} \frac{\partial^{2} G_{1}}{\partial \mathrm{z}^{2}}\right| \frac{1}{2} \frac{d z}{d t}= \pm \gamma_{1}\left|\frac{1}{2} \frac{\partial^{2} G_{1}}{\partial \mathrm{z}^{2}}\right| \frac{\partial^{2} G_{1}}{\partial \mathrm{z}^{2}} z \pm \gamma_{2}\left|\frac{1}{2} \frac{\partial^{2} G_{1}}{\partial \mathrm{z}^{2}}\right| \frac{\partial^{2} G_{1}}{\partial \mathrm{z}^{2}} z+\left|\frac{1}{2} \frac{\partial^{2} G_{1}}{\partial \mathrm{z}^{2}}\right| \frac{1}{2} \frac{\partial^{2} G_{1}}{\partial \mathrm{z}^{2}} z^{2}$.

Substituting $z=\frac{y}{\left|\frac{1 \partial^{2} G_{1}}{z_{a z} z^{2}}\right|}$ we get $\frac{d y}{d t}= \pm \gamma_{1}\left|\frac{1}{2} \frac{\partial^{2} G_{1}}{\partial z^{2}}\right| y \pm \gamma_{2}\left|\frac{1}{2} \frac{\partial^{2} G_{1}}{\partial z^{2}}\right| y+\frac{\frac{1 \partial^{2} G_{1}}{\partial^{2} z^{2}}}{\frac{1}{2}\left|\frac{\partial^{2} G_{1}}{\partial z^{2}}\right|} y^{2}$.

Suppose that $\alpha_{1}=\gamma_{1}\left|\frac{1}{2} \frac{\partial^{2} G_{1}}{\partial \mathrm{z}^{2}}\right|$ and $\alpha_{2}=\gamma_{2}\left|\frac{1}{2} \frac{\partial^{2} G_{1}}{\partial \mathrm{z}^{2}}\right|$. Then we get the normal form

$\dot{y}= \pm \alpha_{1} \mathrm{y} \pm \alpha_{2} \mathrm{y} \pm y^{2}+\mathrm{o}\left(y^{3}\right)$

\subsection{Pitchfork bifurcation}

If two curves of fixed points intersect at the origin in the $\mu-x$ plain and only oneexists in both sides of $\mu=0$, moreover, the other curve of fixed points lays entirely toone side of $\mu=0$, then the origin is called a 
pitchfork bifurcation (PFB) point see [8].

Theorem 3.3. Consider one-dimensional quasilinear DAEs

$$
\mathrm{A}(\mathrm{x}, \mu) \dot{x}=\mathrm{G}(\mathrm{x}, \mu), \quad \mu \in \mathrm{R}^{2}, \mathrm{x} \in \mathrm{R}^{1}
$$

where $G \in R^{3}$ has at $\mu=0$ the eguilibrium $x=0$, and $\frac{\partial G}{\partial x}(0,0,0) \neq 0$. Assume that the following two non-degeneracy conditions are satisfied::

(i) $\frac{\partial^{3} G_{1}}{\partial \mathrm{x}^{3}}(0,0,0) \neq 0$
(ii) $\frac{\partial^{2} G_{1}}{\partial x \partial \mu_{1}}(0,0,0) \neq 0 \& \frac{\partial^{2} G_{1}}{\partial x \partial \mu_{2}}(0,0,0) \neq 0$

then near $(0,0,0),(3.2)$ is topologically equivalent to the one of the following normalforms:

$\dot{y}= \pm \alpha_{1} \mathrm{y} \pm \alpha_{2} \mathrm{y} \pm y^{3}+\mathrm{o}\left(y^{4}\right)(3.10)$

Proof. According to the reduction processes mentioned in (Section 2) DAE will be reduced to ODEs:

$$
\xi=A_{1}(\xi, \mu)^{-1} G_{1}(\xi, \mu)
$$

where $G_{1}(\xi, \mu)$ and $A_{1}(\xi, \mu)^{-1}$ from theorem 2.1 reduced to $G(x, \mu)$ and $A(x, \mu)$. Thenby Taylor expansion about $(0,0,0)$ we have:

$G_{1}\left(\xi, \mu_{1}, \mu_{2}\right)=G_{1}(0,0,0)+\frac{\partial G_{1}}{\partial \xi}(0,0,0) \xi+\frac{\partial G_{1}}{\partial \mu_{1}}(0,0,0) \mu_{1}+\frac{\partial G_{1}}{\partial \mu_{2}}(0,0,0) \mu_{2}+\frac{\partial^{2} G_{1}}{\partial \xi^{2}} \frac{\xi^{2}}{2}+\frac{\partial^{2} G_{1}}{\partial \xi \delta^{\mu}} \xi \mu_{1}+\frac{\partial^{2} G_{1}}{\partial \xi \delta \mu_{2}}+\frac{\partial^{2} G_{1}}{\delta \mu_{1} \partial \mu_{2}} \mu_{1} \mu_{2}+$ $\frac{\partial^{2} G_{1}}{\partial \mu_{1}^{2}} \frac{\mu_{1}^{2}}{2}+\frac{\delta^{2} G_{1}}{\delta \mu_{2}^{2}} \frac{\mu_{2}^{2}}{2}+\frac{\partial^{3} G_{1}}{\partial \xi^{3}} \frac{\xi^{3}}{6}+\cdots$

where.

$G_{1}(0,0,0)=0, \frac{\partial G_{1}}{\partial \xi}(0,0,0)=0, \quad \frac{\partial^{2} G_{1}}{\partial \xi^{2}}=0, \quad \frac{\partial^{3} G_{1}}{\partial \xi^{3}} \neq 0$

From equation (3.11) we get:

$\frac{d z}{d t}=A_{1}(z+\delta, 0,0)^{-1}\left[+\frac{\partial G_{1}}{\partial \mu_{1}} \mu_{1}+\frac{\partial G_{1}}{\partial \mu_{2}} \mu_{2}+\frac{\partial^{2} G_{1}}{\partial \mathrm{z}^{2}} \frac{(\mathrm{z}+\xi)^{2}}{2}+\frac{\partial^{2} G_{1}}{\partial \mathrm{z} \delta \mu_{1}}(\mathrm{z}+\delta) \mu_{1}+\frac{\partial^{2} G_{1}}{\partial \mathrm{z} \delta^{\mu_{2}}}(\mathrm{z}+\delta) \mu_{2}+\right.$

$\left.\frac{\partial^{2} G_{1}}{\delta \mu_{1} \mu_{2}} \mu_{1} \mu_{2}+\frac{\partial^{2} G_{1}}{\partial \mu_{1}{ }^{2}} \frac{\mu_{1}^{2}}{2}+\frac{\delta^{2} G_{1}}{\delta \mu_{2}^{2}} \frac{\mu_{2}^{2}}{2}++\frac{\partial^{3} G_{1}}{\partial \mathrm{z}^{3}} \frac{(\mathrm{z}+\xi)^{3}}{6}+\right] \ldots$

\section{Therefore,}

$\frac{d z}{d t}=A_{1}(z+\delta, 0,0)^{-1}\left[\frac{\partial G_{1}}{\partial \mu_{1}} \mu_{1}+\frac{\partial^{2} G_{1}}{\partial \mu_{1}{ }^{2}} \frac{\mu_{1}{ }^{2}}{2}+\frac{\partial^{2} G_{1}}{\partial \mathrm{z} \delta \mu_{1}} \delta \mu_{1}+\frac{1}{2} \frac{\partial^{2} G_{1}}{\delta \mu_{1} \mu_{2}} \mu_{1} \mu_{2}+\frac{1}{2} \frac{\partial^{2} G_{1}}{\partial \mathrm{z}^{2}} \frac{\delta^{2}}{2}+\frac{1}{2} \frac{\partial^{3} G_{1}}{\partial \mathrm{z}} \frac{\delta^{3}}{36}+z\left(\frac{\partial^{2} G_{1}}{\partial \mathrm{z} \delta \mu_{1}} \delta \mu_{1}+\frac{1}{2} \frac{\partial^{2} G_{1}}{\partial \mathrm{z}^{2}} \delta+\frac{1}{2} \frac{\partial^{3} G_{1}}{\partial \mathrm{z}^{3}} \frac{\delta}{12}\right)+\right.$ $z^{2}\left(\frac{1}{4} \frac{\partial^{2} G_{1}}{\partial \mathrm{z}^{2}} \delta^{2}+\frac{1}{2} \frac{\partial^{3} G_{1}}{\partial \mathrm{z}^{3}} \frac{\delta}{12}\right) \frac{1}{2} \frac{\partial^{2} G_{1}}{\partial \mathrm{z}^{2}} \frac{z^{2}}{2}+\frac{1}{2} \frac{\partial^{3} G_{1}}{\partial \mathrm{z}^{3}} \frac{Z^{3}}{36}+\frac{\partial G_{1}}{\partial \mu_{2}} \mu_{2}+\frac{\partial^{2} G_{1}}{\partial^{2} \mu_{2}} \frac{\mu_{2}}{2}+\frac{\partial^{2} G_{1}}{\partial \mathrm{z} \delta \mu_{2}} \delta \mu_{2}+\frac{1}{2} \frac{\partial^{2} G_{1}}{\delta \mu_{1} \mu_{2}} \mu_{1} \mu_{2}+\frac{1}{2} \frac{\partial^{2} G_{1}}{\partial \mathrm{z}^{2}} \frac{\delta^{2}}{2}+$ $\left.\frac{1}{2} \frac{\partial^{3} G_{1}}{\partial \mathrm{z}^{3}} \frac{\delta^{3}}{36}+z\left(\frac{\partial^{2} G_{1}}{\partial \mathrm{z} \delta \mu_{2}} \delta \mu_{2}+\frac{1}{2} \frac{\partial^{2} G_{1}}{\partial \mathrm{z}^{2}} \delta+\frac{1}{2} \frac{\partial^{3} G_{1}}{\partial \mathrm{z}^{3}} \frac{\delta}{12}\right)+z^{2}\left(\frac{1}{4} \frac{\partial^{2} G_{1}}{\partial \mathrm{z}^{2}} \delta^{2}+\frac{1}{2} \frac{\partial^{3} G_{1}}{\partial \mathrm{z}^{3}} \frac{\delta}{12}\right)+\frac{1}{2} \frac{\partial^{2} G_{1}}{\partial \mathrm{z}} \frac{z^{2}}{2}+\frac{1}{2} \frac{\partial^{3} G_{1}}{\partial \mathrm{z}^{3}} \frac{Z^{3}}{36}\right] .$.

and removing the linear terms $z\left(\frac{\partial^{2} G_{1}}{\partial \mathrm{z} \delta \delta_{1}} \delta \mu_{1}+\frac{\partial^{3} G_{1}}{\partial \mathrm{z}^{3}} \frac{\delta^{2}}{12}\right)$ and $z\left(\frac{\partial^{2} G_{1}}{\partial \mathrm{z} \delta} \delta_{2} \delta \mu_{2}+\frac{\partial^{3} G_{1}}{\partial \mathrm{z}^{3}} \frac{\delta^{2}}{12}\right)$ which isrequired that:

$\delta_{1}(\mu)=-\frac{\frac{\partial^{2} G_{1}}{\partial z \delta^{\mu} 1} \delta}{\frac{\partial^{3} G_{1}}{\partial z^{3}}} \mu_{1}$ and $\delta_{1}(\mu)=-\frac{\frac{\partial^{2} G_{1}}{\partial z \delta^{\mu 2}} \delta}{\frac{\partial^{3} G_{1}}{\partial z^{3}}} \mu_{2}$.

Then the equation becomes:

$\frac{d z}{d t}=\beta_{1} \frac{\partial^{3} G_{1}}{\partial \mathrm{z}^{3}} z+\beta_{2} \frac{\partial^{3} G_{1}}{\partial \mathrm{z}^{3}} z+\frac{\partial^{3} G_{1}}{\partial \mathrm{z}^{3}} z^{3}$ 


\section{Where}

$\beta_{1}=\frac{\partial^{2} G_{1}}{\partial \mathrm{z} \delta \mu_{1}} \delta \mu_{1}+\frac{1}{2} \frac{\partial^{2} G_{1}}{\partial z^{2}} \delta++\frac{\partial^{3} G_{1}}{\partial z^{3}} \frac{\delta^{2}}{12}$

$\beta_{2}=\frac{\partial^{2} G_{1}}{\partial \mathrm{z} \delta \mu_{2}} \delta \mu_{2}+\frac{1}{2} \frac{\partial^{2} G_{1}}{\partial \mathrm{z}^{\mathrm{z}}} \delta++\frac{\partial^{3} G_{1}}{\partial \mathrm{z}^{3}} \frac{\delta^{2}}{12}$.

Now consider as a new parameter $\beta=\left(\beta_{1}(\mu), \beta_{2}(\mu)\right)$ and we have $A_{1}(z+\delta, 0,0)^{-1}$

is independent of $\xi$ and since $\frac{\partial^{2} G_{1}}{\partial \mathrm{z} \delta \mu_{1}} \neq 0 \quad \& \frac{\partial^{2} G_{1}}{\partial \mathrm{z} \delta \mu_{2}} \neq 0$ we can neglecting terms with $\mu_{1}^{2} \& \mu_{2}^{2}$ respectively then we have:

$\beta_{1} \approx \frac{\partial^{2} G_{1}}{\partial \mathrm{z} \delta \mu_{1}} \mu_{1} \beta_{2} \approx \frac{\partial^{2} G_{1}}{\partial \mathrm{z} \delta \mu_{1}} \mu_{2}$.

So for $\mu_{1}>0, \frac{\partial^{2} G_{1}}{\partial \mathrm{z} \delta \mu_{1}}>0, \beta_{1} \quad$ is increasing and $\mu_{2}>0, \& \frac{\partial^{2} G_{1}}{\partial \mathrm{z} \delta \mu_{2}}>0, \beta_{2}$

is increasing, but if $\frac{\partial^{2} G_{1}}{\partial \mathrm{z} \delta \mu_{1}}<0 \& \frac{\partial^{2} G_{1}}{\partial \mathrm{z} \delta \mu_{2}}$ then $\beta_{1}, \beta_{2}$ are decreasing when $\mu_{1}<0, \mu_{1}<0$ resp. Now let the following assumption

$\gamma_{1}=\left\{\begin{array}{l}\beta_{1} i f \frac{\partial^{2} G_{1}}{\partial \mathrm{z} \delta \mu_{1}}>0 \\ \beta_{1} i f \frac{\partial^{2} G_{1}}{\partial \mathrm{z} \delta \mu_{1}}<0\end{array} \gamma_{1}=\left\{\begin{array}{l}\beta_{2} i f \frac{\partial^{2} G_{1}}{\partial \mathrm{z} \delta \mu_{2}}>0 \\ \beta_{2} \text { if } \frac{\partial^{2} G_{1}}{\partial \mathrm{z} \delta \mu_{2}}<0\end{array}\right.\right.$

Then the equation is:

$\frac{d z}{d t} \pm \gamma_{1} \frac{\partial^{3} G_{1}}{\partial z^{3}} z^{+} \gamma_{2} \frac{\partial^{3} G_{1}}{\partial z^{3}} z \pm \frac{1}{2} \frac{\partial^{3} G_{1}}{\partial z^{3}} z^{3}$.

Next assume $y=\left|\frac{1}{2} \frac{\partial^{3} G_{1}}{\partial z^{3}}\right| z$ then we have:

$\frac{d y}{d t}=\left|\frac{1}{2} \frac{\partial^{3} G_{1}}{\partial \mathrm{z}^{2}}\right| \frac{1}{2} \frac{d z}{d t}= \pm \gamma_{1}\left|\frac{1}{2} \frac{\partial^{3} G_{1}}{\partial \mathrm{z}^{2}}\right| \frac{\partial^{3} G_{1}}{\partial \mathrm{z}^{3}} z \pm \gamma_{2}\left|\frac{1}{2} \frac{\partial^{3} G_{1}}{\partial \mathrm{z}^{2}}\right| \frac{\partial^{3} G_{1}}{\partial \mathrm{z}^{3}} z+\left|\frac{1}{2} \frac{\partial^{3} G_{1}}{\partial \mathrm{z}^{3}}\right| \frac{1}{2} \frac{\partial^{3} G_{1}}{\partial \mathrm{z}^{3}} z^{3}$.

Substituting $\mathrm{z}=\frac{y}{\left|\frac{1 \partial^{3} G_{1}}{2 \partial^{3} z^{3}}\right|}$ we get :

$\frac{d y}{d t}= \pm \gamma_{1} \frac{\frac{1 \partial^{3} G_{1}}{2 \partial^{3}}}{\frac{1}{2}\left|\frac{\partial^{3} G_{1}}{\partial z^{3}}\right|} y \pm \gamma_{2} \frac{\frac{1 \partial^{3} G_{1}}{2 z^{3}}}{\frac{1}{2}\left|\frac{\partial^{3} G_{1}}{\partial z^{3}}\right|} y+\frac{\frac{1 \partial^{3} G_{1}}{2 \partial^{3}}}{\frac{1}{2}\left|\frac{\partial^{3} G_{1}}{\partial z^{3}}\right|} y^{3}$

Suppose that $\alpha_{1}=\gamma_{1}\left|\frac{1}{2} \frac{\partial^{3} G_{1}}{\partial \mathrm{z}^{3}}\right|$ and $\alpha_{2}=\gamma_{2}\left|\frac{1}{2} \frac{\partial^{3} G_{1}}{\partial \mathrm{z}^{3}}\right|$ Then we get the normal form

$\dot{y}= \pm \alpha_{1} \mathrm{y} \pm \alpha_{2} \mathrm{y} \pm y^{3}+\mathrm{o}\left(y^{4}\right)$

\section{Applications OF DAEs}

There are three types of one-zero-eigenvalue bifurcations: saddle-node, trans-criticaland pitchfork bifurcation.

Each one of them satisfies different genericity conditions; their bifurcation diagrams are also different.

Without loss of generality let us assumethat $(\mathrm{x}, \mu)=(0,0)$ is point of one-dimensional parameterized dynamical system:

$A(\mu, x) \dot{x}=G(\mu, x)$

$\mu \in \mathrm{R}^{\mathrm{n}}, \mathrm{x} \in \mathrm{R}^{\mathrm{n}}$

Now, for (4.1) to undergo a one-zero-eigenvalue bifurcation at $(0,0)$, the following

conditions should be satisfied: 


$$
\mathrm{G}(0,0)=0, \mathrm{G}_{\mathbf{X}}(0,0)=0 \text {. }
$$

These conditions guarantee that the fixed point $(0,0)$ is not hyperbolic.The natural environment for this kind of work are the computer algebra systems likeMaple and Mathematica.

Their impact on dynamical systems studies is due to thefact that many calculations are too tedious for manual work, but do not ch allengethe computer resources .

In this paper we present algorithms for symbolical study ofone parameter local bifurcations in guasilinear DAEs of equilibrium points an $\mathrm{d}$ discusstheir implementation in Maple.

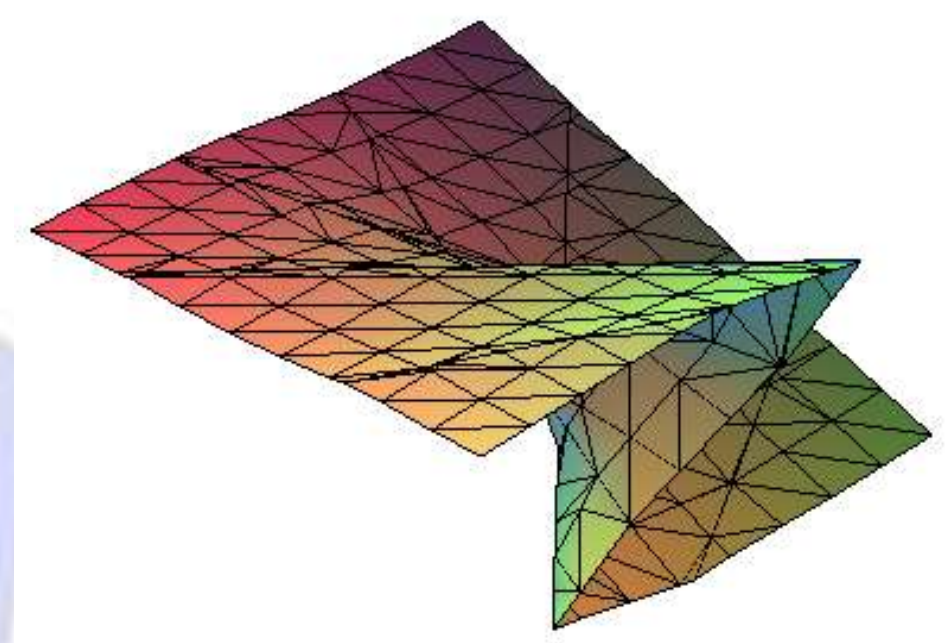

Figure 3: The normal form of a Pitchfork bifurcation, where $\mathrm{r}$ ranges from $\pi$ to $-\pi$ using maple

\section{References}

[1] Thompson, J M T and H B stewart ( 1986), Nonlinear Dynamics and Chaos (J)Wiley, Chichester). tischendorf, C

( 1995), Feasibility and stability behaviour of theBDF applied to index2 differential algebraic equations, Z Angew Math Mec h 75,927-946.

[2] Jepson, A. and Spence, A. [1985]. Fold in solutions of two parameter systems and their calculation. Part 1.Siam J. Numer. Anal. Vol.2. No.2. 347-368.

[3] Keller,H. [1977],Numerical solution of bifurcation and nonlinear eigenvalue problems, in P. Rabinowitz,ed.,Applications of Bifurcation Theory, Academic Press,NewYork,pp. 359384.

[4] Crandall, M. and Rabinowitz,P.[1973]. Bifurcation, perturbation of simple eigen values, and linearized stability. Arch. Rational.Mech. Anal.,52, 161-180.

[5] RabierP. J. and Rheinboldt W. C., Theoretical and numerical analysis of

differential-algebraic equations, in P. G. Ciarlet et al. (eds.), Handbook of Nu-merical Analysis, Vol. VIII, pp. 183540, North Holland/Elsevier, 2002.

[6] Oswaldo Rio Branco de Oliveira Implicit Function Theorems, Calculus of Vector Functions, Differential Calculus, Funct ons of Several Variables,26B10, 26B12,97140, 97160.

[7] Lawrence Perko, Differential Equations and Dynamical system. Springer-Verlay,New York, 3rd Edition, 2001.

[8] Stephen, Wiggins. Introduction to Applied Nonlinear Dynamical Systems and Chaos. Springer-Verlay, New York, 1990. 